\title{
Improving birth outcomes for women who are substance using or have mental illness: a Canadian cohort study comparing antenatal midwifery and physician models of care for women of low socioeconomic position
}

\author{
Daphne N. McRae ${ }^{1 *}$ (D), Nazeem Muhajarine ${ }^{1}$ and Patricia A. Janssen ${ }^{2}$
}

\begin{abstract}
Background: Some observational studies have shown improved birth outcomes for women of low socioeconomic position (SEP) receiving antenatal midwifery versus physician care. To understand for whom and under what circumstances midwifery care is associated with better birth outcomes we examined whether psychosocial risk including substance use, mental illness, social assistance, residence in a neighbourhood of low/moderate SEP, and teen maternal age modified the association between model of care (midwifery versus physician) and small-forgestational-age (SGA) or preterm birth (PTB) for women of low SEP.

Methods: For this retrospective cohort study, maternity data from the British Columbia Perinatal Data Registry were linked with Medical Services Plan billing data. We report adjusted odds ratios (aORs) and 95\% confidence intervals (Cls) for SGA birth (< the 10th percentile) and PTB (<37 weeks' completed gestation). For tests of interaction between antenatal models of care and psychosocial risk, $p$-values $<0.10$ were considered statistically significant. Women were eligible for inclusion if they were residents of British Columbia, Canada, carried a singleton fetus, had low to moderate medical/obstetric risk, birthed between April 1, 2008 and Dec. 31, 2012, and received a health insurance subsidy $(n=33,937)$.

Results: Midwifery versus obstetrician patients had lower odds of PTB. The difference was 31\% larger among substance users (aOR 0.24, 95\% Cl: 0.11-0.54) compared to non-substance users (aOR 0.55, 95\% Cl: 0.45-0.68). Additionally, there was a 34\% statistically significant absolute difference in odds of PTB for midwifery versus obstetrician patients with both mental illness and substance use (aOR 0.18, 95\% Cl: 0.06-0.55) compared to women with neither mental illness nor substance use (aOR 0.52, 95\% Cl: 0.41-.66). Results demonstrated a consistent association between midwifery versus physician care and lower odds of SGA, yet effects were not statistically significantly different for women with higher or lower psychosocial risk.

\footnotetext{
* Correspondence: daphne.mcrae@usask.ca

${ }^{1}$ Department of Community Health and Epidemiology, University of

Saskatchewan, Box 7 Health Science Building 107 Wiggins Road, Saskatoon

SK S7N 5E5, Canada

Full list of author information is available at the end of the article
}

(c) The Author(s). 2019 Open Access This article is distributed under the terms of the Creative Commons Attribution 4.0 International License (http://creativecommons.org/licenses/by/4.0/), which permits unrestricted use, distribution, and reproduction in any medium, provided you give appropriate credit to the original author(s) and the source, provide a link to the Creative Commons license, and indicate if changes were made. The Creative Commons Public Domain Dedication waiver (http://creativecommons.org/publicdomain/zero/1.0/) applies to the data made available in this article, unless otherwise stated. 
(Continued from previous page)

Conclusion: Among low SEP women in British Columbia, Canada, antenatal midwifery compared to obstetrician care was associated with reduced odds of PTB. Odds were lower among women with substance use, and mental illness and substance use, than among women without these risk factors.

Keywords: Midwifery, Mental health, Substance use, Preterm birth, Small-for-gestational-age, Socioeconomic position, Health services research

\section{Introduction}

A Cochrane Review published in 2016, involving eight trials $(n=13,238)$ in high income countries, demonstrated a $24 \%$ reduction in preterm birth (risk ratio: $0.76,95 \%$ confidence interval $(\mathrm{CI}): 0.64,0.91$ ) for women randomized to receive perinatal care from a single midwife or a small call group compared to women in other models of care (e.g. physician-led, or midwifery-physician models) [1]. After examining the review, the World Health Organization reported that the evidence in support of this finding is of moderate certainty [2]. In response, the WHO has recommended access to "well-functioning midwifery programs" ([2], p89) as a health systems' intervention for improving utilization and quality of antenatal care.

Some observational studies specifically focusing on women of low socioeconomic position (SEP) have also demonstrated better birth outcomes for midwives' versus physicians' patients [3]. We recently published a retrospective cohort study from British Columbia (BC), Canada, demonstrating a 29 to $41 \%$ reduction in odds of small-for-gestational-age (SGA) birth and a 26 to $47 \%$ reduction in odds of preterm birth (PTB) for midwifery versus general practitioner $(\mathrm{GP})$ or obstetrician $(\mathrm{OB})$ patients of low SEP who had low to moderate perinatal risk [4]. The aim of this current analysis is to understand for whom and under what circumstances midwifery care is associated with better birth outcomes. Therefore, we examined whether psychosocial risk including substance use, mental illness, social assistance, residence in a neighbourhood of low/moderate socioeconomic position, and teen maternal age modified the association between model of care (midwifery versus physician) and small-for-gestational-age (SGA) or preterm birth (PTB) for women of low SEP.

\section{Methods}

This section provides a summary of the previously published, full study protocol [4].

\section{Setting}

In $\mathrm{BC}$, women self-select their maternity provider (generally GPs, OBs, or midwives) subject to availability. Women with no perinatal complications or low to moderate pregnancy risk (as defined by the BC College of Midwives' guidelines [5]) are eligible to receive care from any type of registered provider, with fees paid by the provincial Medical Services Plan (MSP). Midwives are required to consult with a physician (usually an $\mathrm{OB}$ ) for moderate pregnancy complications and to transfer care to an OB for high-risk complications [5]. Midwifery care in Canada is based on a relational model where care is provided by a single midwife or small pool of midwives known to a client and accessible by phone $24 \mathrm{~h}$ a day [6]. The midwifery model emphasizes holistic care, continuity of care provider, and informed choice (particularly concerning medical interventions and birth location). Midwives have a capped annual caseload and are paid per full or partial trimester of care [6] enabling lengthy antenatal visits (30 to $60 \mathrm{~min}$ on average [7]).

\section{Study design}

For this retrospective, population-level cohort study we assessed the association between antenatal models of care and SGA and PTB for low SEP women with and without specific psychosocial risk factors. Model of care was determined by linking women's maternity records to practitioners' MSP billing records (billing records indicate the type(s) of practitioner(s) involved in antenatal care). Maternity data, including data on mental health, substance use, and teen maternal age, were obtained from the BC Perinatal Data Registry (PDR) [8], which includes data from hospital and home birth records as well as International Statistical Classification of Diseases, Tenth Revision, Canada (ICD-10-CA) codes from the Canadian Institutes of Health Information Discharge Abstract Database. Approximately 99\% of all BC births are recorded in the PDR [9] with a chart re-abstraction study showing high validity on key surveillance variables [10].

PDR data were linked to MSP billing data [11] to determine if women were of low SEP and to identify women receiving social assistance (public financial aid for low income). Low SEP was operationalized as receipt of a regular MSP health insurance subsidy during the year of delivery [11]. Subsidy assistance is offered to low income individuals based on their previous year's household income (e.g. a family of three earning $\$ 28,000$ or less in $2008 / 2009$ or $\$ 30,000$ or less in 2010-2012 would have been eligible for a health insurance subsidy) [11]. Women receiving social assistance have their health 
insurance premiums waived and this information is also recorded in the MSP billing data.

Maternity and billing data were linked with neighbourhood demographic data provided by Population Data BC [12], and Local Health Area (LHA) socioeconomic and income inequality rankings publicly available from BC's Division of Statistics [13, 14]. Variables from these sources were tested as modifying (neighbourhood SEP) or confounding factors (LHA socioeconomic rank and LHA income inequality).

\section{Study sample}

Our study included pregnant, low SEP women who were residents of $\mathrm{BC}$, received antenatal midwifery, GP, or $\mathrm{OB}$ care, carried a singleton fetus, were eligible for midwifery care throughout the antenatal period (having no or low to moderate medical/obstetric risk according to midwifery guidelines [5]), birthed between April 1, 2008 and Dec. 31, 2012, received an MSP health insurance subsidy, and were not registered Status Indian ${ }^{1}(n=33$, 937). ('Status Indian' is a legal term referring to the Indigenous identity of people registered under the Canadian government's Indian Act and eligible for certain government benefits and services [15]). Status Indian women were excluded from the study because they had their health insurance premiums paid by Health Canada and therefore were not eligible for an MSP health insurance subsidy.

Moderate medical and/or obstetric risk (defined in Table 1) included conditions which would require a midwife to consult with an $\mathrm{OB}$ yet allow a midwife to retain the primary care provider role. As OB patients can have higher pregnancy risk than midwifery clients we excluded all physicians' patients who had antenatal conditions rendering them ineligible for midwifery care. Likewise, we excluded all midwifery clients who required a transfer to $\mathrm{OB}$ care during the antenatal period. Only eligible cases with complete data were included in the analyses $(99.79 \%$ of the cases for SGA and $99.87 \%$ for PTB).

\section{Outcomes}

Our primary outcome was SGA birth (<10th percentile) according to Kierans' et al. sex-specific birth weight references. The reference tables are applicable for singleton, live-born infants between 20 and 44 weeks' completed gestation. They specify threshold values for infant weight by gestational age which equate to less than the 10th percentile of provincial birth weight distributions.

\footnotetext{
${ }^{1}$ Indigenous and Northern Affairs Canada https://www.aadnc-aandc.gc. ca/eng/1100100032463/1100100032464\#chp1, and Statistics Canada http://www23.statcan.gc.ca/imdb/p3Var.pl?Function=DEC\&Id=42932 specify the proper use of the term 'Status Indian', as applied in our study.
}

This birth weight reference is the most appropriate tool for classifying SGA birth in our study as it was constructed using population-based birth weight distributions from BC between 1981 and 2000, and was approved for use in BC hospitals as of 2004 [16]. PTB was defined as birth less than 37 weeks' completed gestation.

\section{Statistical analyses}

Data was analyzed using multivariable, logistic regression models and a Generalized Estimating Equation (GEE) approach to account for clustering of effects by siblings and by community [17]. Women self-selected their model of care therefore those who knew they had higher pregnancy risk (within the low to moderate risk spectrum) could have chosen OB care more often resulting in selection bias. To address this concern we conducted two sensitivity analyses. First, we controlled for select antepartum morbidities (see definition in Table 1) which could be associated with medical conditions, such as uterine anomaly, undocumented in the PDR. Second, we excluded all patients with prior medical or obstetric risk to assess if differences in severity of known risk factors between practitioner-types could explain the observed associations.

To investigate if psychosocial risk (substance use, mental illness, social assistance, low/moderate neighbourhood SEP, teen maternal age) modified the association between model of care and SGA or PTB, interaction terms comprised of model of care multiplied by each type of risk were included in the GEE models. The effect of combined psychosocial risk was tested using two-way interactions, e.g. "model of care $\mathrm{x}$ mental illness and substance use" (defined as women with both versus neither). We report adjusted odds ratios (aORs) and $95 \%$ CIs for the association between model of care and SGA/PTB. For tests of interaction we report $p$ values. For interactions we considered $p<0.10$ as statistically significant because the aim was to identify clinically important differences between subgroups, if any, within the larger sample and therefore maximize possibilities for tailored interventions [18]. Our assessments for effect modification were based on the statistical significance of the interactions, the consistency between the direction of the main effect estimates and subgroup effects, patterns of effect for different types of psychosocial risk, and significance of the subgroup aORs [19]. SAS Enterprise 7.1 was utilized for all data analyses [20].

\section{Results}

There were 3397 midwifery, 25,784 GP and $4756 \mathrm{OB}$ pregnancies included in the analyses (Table 1). Midwifery clients had significantly higher prevalence of mental 
Table 1 Frequencies and proportions of maternal characteristics by antenatal model of care, British Columbia, April 1, 2008December 31, $2012(n=33,937)$

\begin{tabular}{|c|c|c|c|}
\hline \multirow[t]{2}{*}{ Maternal Characteristics } & \multicolumn{3}{|c|}{ Antenatal Model of Care } \\
\hline & $\mathrm{MW} n=3397 n(\%)$ & GP $n=25,784 n(\%)$ & OB $n=4756 n(\%)$ \\
\hline \multicolumn{4}{|l|}{ Age (yrs.) } \\
\hline $14-19$ & $117(3.44)$ & $2587(10.03)$ & $208(4.37)$ \\
\hline $20-24$ & $644(18.96)$ & $8366(32.45)$ & $841(17.68)$ \\
\hline $25-29$ & $1161(34.18)$ & $7716(29.93)$ & $1371(28.83)$ \\
\hline $30-34$ & $987(29.06)$ & 4559 (17.68) & $1247(26.22)$ \\
\hline $35-39$ & $415(12.22)$ & $2113(8.20)$ & $808(16.99)$ \\
\hline$>40$ & $73(2.15)$ & $443(1.72)$ & $281(5.91)$ \\
\hline \multicolumn{4}{|l|}{ Parity $^{a}$} \\
\hline Nullipara & $1607(47.31)$ & $13,148(50.99)$ & $2134(44.87)$ \\
\hline Multipara & $1790(52.69)$ & $12,636(49.01)$ & $2621(55.11)$ \\
\hline Medical risk ${ }^{\mathrm{b}, \mathrm{c}}$ & $11(0.32)$ & $263(1.02)$ & $90(1.89)$ \\
\hline Prior obstetric risk ${ }^{b, d}$ & $87(2.56)$ & $999(3.87)$ & $280(5.89)$ \\
\hline Mental illness ${ }^{b, e}$ & $1012(29.79)$ & $4922(19.09)$ & $545(11.46)$ \\
\hline Receiving social assistance ${ }^{b}$ & $239(7.04)$ & $3623(14.05)$ & $535(11.25)$ \\
\hline \multicolumn{4}{|l|}{ Pre-pregnancy Body Mass Index ${ }^{f}$} \\
\hline Underweight & $156(4.59)$ & $1281(4.97)$ & $277(5.82)$ \\
\hline Normal & $1854(54.58)$ & $9616(37.29)$ & $1652(34.74)$ \\
\hline Overweight & $511(15.04)$ & 3554 (13.78) & $528(11.10)$ \\
\hline Obese & $243(7.15)$ & $2340(9.08)$ & $327(6.88)$ \\
\hline Unknown & $633(18.63)$ & $8993(34.88)$ & $1972(41.46)$ \\
\hline \multicolumn{4}{|l|}{ Smoking status } \\
\hline Never & $915(26.94)$ & $5189(20.12)$ & $1325(27.86)$ \\
\hline Former & $548(16.13)$ & $3456(13.40)$ & $299(6.29)$ \\
\hline Current & $336(9.89)$ & $5540(21.49)$ & $459(9.65)$ \\
\hline Unknown & $1598(47.04)$ & $11,599(44.99)$ & $2673(56.20)$ \\
\hline Substance use in pregnancy ${ }^{\mathrm{b}, \mathrm{g}}$ & $153(4.50)$ & $2281(8.85)$ & $182(3.83)$ \\
\hline Alcohol identified as a risk ${ }^{\mathrm{b}}$ & $47(1.38)$ & $753(2.92)$ & $31(0.65)$ \\
\hline Antepartum morbidity ${ }^{b, h}$ & $243(7.15)$ & $3863(14.98)$ & $1189(25.00)$ \\
\hline \multicolumn{4}{|l|}{ Delivery year } \\
\hline 2008 (as of April 1) & 419 (12.33) & $4495(17.43)$ & $711(14.95)$ \\
\hline 2009 & $606(17.84)$ & $5640(21.87)$ & $910(19.13)$ \\
\hline 2010 & $694(20.43)$ & $5371(20.83)$ & $1000(21.03)$ \\
\hline 2011 & $796(23.43)$ & $5337(20.70)$ & $1014(21.32)$ \\
\hline 2012 & $882(25.96)$ & $4941(19.16)$ & $1121(23.57)$ \\
\hline \multicolumn{4}{|l|}{ Neighbourhood SEP' } \\
\hline High & $450(13.25)$ & $2846(11.04)$ & $377(7.93)$ \\
\hline Low/Medium & $2947(86.75)$ & $22,938(88.96)$ & $4379(92.07)$ \\
\hline \multicolumn{4}{|l|}{ LHA Socioeconomic Rank $k^{j}$} \\
\hline High (Best) & $1858(54.70)$ & $7314(28.37)$ & $2327(48.93)$ \\
\hline Medium & 1118 (32.91) & $12,774(49.54)$ & 1897 (39.89) \\
\hline Low & $412(12.13)$ & $5641(21.88)$ & $487(10.24)$ \\
\hline Unknown & $9(0.26)$ & $55(0.21)$ & $45(0.95)$ \\
\hline
\end{tabular}

LHA Income Inequality Rank ${ }^{k}$ 
Table 1 Frequencies and proportions of maternal characteristics by antenatal model of care, British Columbia, April 1, 2008December 31, $2012(n=33,937)$ (Continued)

\begin{tabular}{llll}
\hline Maternal Characteristics & \multicolumn{3}{l}{ Antenatal Model of Care } \\
\cline { 2 - 4 } & MW $n=3397 n(\%)$ & GP $n=25,784 n(\%)$ & OB $n=4756 n(\%)$ \\
\hline High (Worst) & $1188(34.97)$ & $5776(22.40)$ & $2419(50.86)$ \\
Medium & $1717(50.54)$ & $14,829(57.51)$ & $1999(42.03)$ \\
Low & $483(14.22)$ & $5133(19.91)$ & $318(6.69)$ \\
Unknown & $9(0.26)$ & $46(0.18)$ & $20(0.42)$ \\
Northern residence & $92(2.71)$ & $3426(13.29)$ & $205(4.31)$ \\
\hline
\end{tabular}

Abbreviations: GP general practitioner, $M W$ midwife, $O B$ obstetrician, SEP socioeconomic position

${ }^{a}$ Missing cases amount to 5 or less

"Values represent cases classified as "Yes", the remainder of the cases were classified as "No", "Unknown", or were undocumented

"Included maternal disease of the respiratory or digestive system, and endocrine, nutritional, or metabolic disease

${ }^{d}$ Included women with at least one of the following conditions in past pregnancy: infant with major congenital anomaly, neonatal death, stillbirth, or one preterm delivery

e Included any of the following diagnoses prior to, or during the current pregnancy: anxiety disorder, depression, postpartum depression, bipolar disorder, other/unknown (including schizophrenic, mood, and psychotic disorders)

fClassified according to Health Canada's guidelines [34]

${ }^{9}$ Heroin/opioids, cocaine, methadone, solvents, marijuana, or other/unknown drugs used at any time during pregnancy, prescription or other drug use identified as a risk at any time during pregnancy

hIncluded pregnancy induced hypertension, gestational diabetes (whether or not insulin dependent), anemia, intrauterine growth restriction, viral disease, infection and parasitic disease, placenta previa without hemorrhage, polyhydramnios or oligohydramnios, antepartum hemorrhage $>20$ weeks, sexually transmitted infection or HIV, or premature separation of the placenta

'Neighbourhood income quintiles were classified as low/medium (quintiles 1-4) versus high (quintile 5) [12]

${ }^{\mathrm{j}}$ Calculated by the province of BC's Statistics Division (BC Stats), based on a range of social determinants of health reflecting area-level economic and social processes, and policy decisions [14]

${ }^{\mathrm{k}}$ Calculated by BC Stats [14]

'At the time of delivery, normal residence in BC's Northern Health Authority

illness $(29.79 \%)$ compared to GP (19.09\%) or OB patients (11.46\%). Depression was the most frequent mental health condition reported. The prevalence of depression, as indicated in the maternal record, was much greater among midwives' clients (18.55\%) than GPs' $(12.60 \%)$ or OBs' patients (7.44\%). Substance use was more frequently reported among GPs' patients (8.85\%) than either midwives' $(4.50 \%)$ or OBs' patients (3.83\%). Likewise, there was a greater proportion of teen mothers attended during the antenatal period by GPs $(10.03 \%)$ than by OBs $(4.37 \%)$ or midwives (3.44\%). GPs also cared for a greater proportion of women receiving social assistance $(14.05 \%)$ than either OBs (11.25\%) or midwives (7.04\%). Most women lived in low or middle SEP neighbourhoods, with a slightly larger proportion of midwifery clients living in high SEP neighbourhoods (13.25\% versus $11.04 \%$ for GPs' and 7.93\% for OBs' patients).

\section{Small-for-gestational-age birth}

Of the 33,866 eligible births included in the analysis, 2378 (7.02\%) were SGA (Table 2). On average, women of low SEP receiving antenatal midwifery care, compared to GP care, had lower adjusted odds of SGA birth (aOR 0.73, 95\% CI: 0.62-0.86). (Model adjusted for maternal age, parity, pre-pregnancy BMI, infant sex, smoking status, substance use, mental illness/disorder, and Local Health Area socioeconomic rank). Likewise, midwifery versus $\mathrm{OB}$ patients were less likely to have an SGA birth (aOR 0.60, 95\% CI: $0.49-0.72$ ) as were GP versus OB patients (aOR 0.82: 95\% CI: 0.73-0.93). When adjusting for select antepartum morbidities (see definition in Table 1) and when excluding patients with prior obstetric or medical risk to control for the possibility of $\mathrm{OB}$ patients having systematically higher pregnancy risk on the low to moderate risk spectrum, midwifery clients continued to have significantly more favourable birth outcomes than physicians' patients (see results of the sensitivity analyses in Additional file 1).

Adjusted odds of SGA for midwifery versus GP or OB patients were lower for those with mental illness, substance use, or mental illness and substance use, compared to women without these psychosocial risk factors. Yet the difference in effect estimates were not statistically significant for those with higher versus lower psychosocial risk. For example, both substance users $(\mathrm{aOR}$ 0.41, 95\% CI: 0.18-0.93) and non-substance users (aOR 0.75, 95\% CI: 0.63-0.89), had significantly lower odds of $S G A$ if in the care of midwives versus GPs (Table 2), however odds ratios were not significantly different $(p=0.16)$ across the substance user and non-user groups.

Likewise for midwifery versus OB patients, odds of SGA were $31 \%$ lower among substance users compared to non-substance users (aOR 0.31, 95\% CI: 0.12-0.78 versus aOR $0.62,95 \% \mathrm{CI}: 0.51-0.75)$, but tests of interaction did not indicate a significant difference in odds by substance user versus non-user strata $(p=0.15)$. When 
Table 2 Frequencies and adjusted odds ratios for small-for-gestational-age birth by antenatal model of care and psychosocial risk characteristics, British Columbia, April 1, 2008-December 31, 2012

\begin{tabular}{|c|c|c|c|c|c|c|}
\hline \multicolumn{7}{|c|}{ Small-for-gestational-age birth by model of care } \\
\hline & MW $n=3391$ & $G P n=25,733$ & $\mathrm{OB} n=4742$ & MW vs. GP & MW vs. OB & GP vs. OB \\
\hline & $n(\%)$ & $n(\%)$ & $n(\%)$ & $\mathrm{aOR}(95 \% \mathrm{Cl})$ & $\mathrm{aOR}(95 \% \mathrm{Cl})$ & $\mathrm{aOR}(95 \% \mathrm{Cl})$ \\
\hline \multicolumn{7}{|l|}{ Mental illness ${ }^{a}$} \\
\hline Yes & $42 / 1011(4.15)$ & $340 / 4912(6.92)$ & $45 / 545(8.26)$ & $0.64(0.46-0.89)$ & $0.49(0.32-0.77)$ & $0.77(0.55-1.08)$ \\
\hline No & $129 / 2380(5.42)$ & $1461 / 20,821(7.02)$ & $361 / 4197(8.60)$ & $0.76(0.63-0.92)$ & $0.63(0.51-0.78)$ & $0.83(0.73-0.94)$ \\
\hline \multicolumn{7}{|l|}{ Substance use $\mathrm{b}^{\mathrm{b}}$} \\
\hline Yes & $6 / 152(3.95)$ & $222 / 2275(9.76)$ & 23/182 (12.64) & $0.41(0.18-0.93)$ & $0.31(0.12-0.78)$ & $0.75(0.46-1.21)$ \\
\hline No & $165 / 3239(5.09)$ & $1579 / 23,458(6.73)$ & $383 / 4560(8.40)$ & $0.75(0.63-0.89)$ & $0.62(0.51-0.75)$ & $0.83(0.73-0.94)$ \\
\hline \multicolumn{7}{|l|}{ Teen mother } \\
\hline Yes (14-19yrs.) & $8 / 117(6.84)$ & $181 / 2582(7.01)$ & 21/205 (10.24) & $0.94(0.45-1.98)$ & $0.56(0.24-1.34)$ & $0.60(0.36-0.99)$ \\
\hline No (25-29yrs.) & $55 / 1159(4.75)$ & $537 / 7697(6.98)$ & $126 / 1370(9.20)$ & $0.71(0.53-0.94)$ & $0.55(0.39-0.76)$ & $0.78(0.63-0.96)$ \\
\hline \multicolumn{7}{|c|}{ Mental illness, substance use } \\
\hline Both & 5 or less $/ 95^{c}$ & $90 / 927(9.71)$ & 10/76 (13.16) & $0.33(0.10-1.07)$ & $0.22(0.06-0.87)$ & $0.68(0.32-1.43)$ \\
\hline Neither & $126 / 2322(5.43)$ & $1324 / 19,424(6.82)$ & $348 / 4086(8.52)$ & $0.78(0.64-0.94)$ & $0.64(0.51-0.79)$ & $0.82(0.72-0.94)$ \\
\hline
\end{tabular}

Abbreviations: $M W$ midwife, GP general practitioner, $O B$ obstetrician, $O R$ odds ratio, $\mathrm{Cl}$ confidence interval

${ }^{a}$ Mental illness included any of the following diagnoses prior to, or during the current pregnancy: anxiety disorder, depression, postpartum depression, bipolar

disorder, other/unknown (including schizophrenic, mood, and psychotic disorders). Aside from mild anxiety or depression, a physician would diagnose mental illness

${ }^{\mathrm{b}}$ Substance use included any indication in the medical record of heroin/opioids, cocaine, methadone, solvents, marijuana or other/unknown drugs used by the mother at any time during pregnancy, as well as prescription or other drug use identified as a risk by the provider

'Percentage suppressed due to small cell size

*Outcomes had statistically significant tests of interaction $(p<0.10)$ comparing difference of effect across strata (yes vs. no)

Models adjusted for all variables listed except stratifying variables: maternal age, parity, pre-pregnancy BMI, infant sex, smoking status, substance use, mental illness/disorder, and Local Health Area (LHA) socioeconomic rank

Odds ratios based on 2378 births with SGA and 33,866 total births with no missing information for this analysis

testing the modifying effect of mental illness (presence versus absence), teen maternal age (14-19 versus 2529 ), and mental illness and substance use (both versus neither) on SGA by model of care there was no evidence of effect modification.

\section{Preterm birth}

Overall, preterm birth occurred in $6.43 \%(n=2178)$ of the eligible study sample $(n=33,893)$ (Table 3$)$. The adjusted odds of PTB was statistically significantly smaller for woman of low SEP receiving antenatal care from midwives versus GPs (aOR 0.79, 95\% CI: 0.66-0.94) and midwives versus OBs (aOR 0.53, 95\% CI: 0.43-0.64). Models were adjusted for maternal age, medical risk, prior obstetric risk, pre-pregnancy BMI, infant sex, receipt of social assistance, smoking status, substance use, mental illness/disorder, neighbourhood SEP, Local Health Area socioeconomic rank, and northern residence. On average, GP patients were also less likely to have a PTB than OB patients (aOR 0.67, 95\% CI: $0.59-$ 0.75).

When assessing residual confounding by excluding women with prior obstetric risk or medical risk from the analysis, the odds of PTB remained significantly lower for midwives' versus physicians' patients (see results in Additional file 1). This provides evidence of an effect by model of care, independent of perinatal risk. When controlling for morbidity arising during the antenatal period, results demonstrated statistically significant reductions in odds of PTB for midwives' versus OBs' patients and GPs' versus OBs' patients but no significant difference in odds for midwives' versus GPs' patients. Slightly higher prevalence of antenatal morbidity for GPs' versus midwives' patients may help to explain the lower odds of PTB midwives' clients experienced. However when we conducted the same sensitivity analysis with a larger sample of low SEP women $(n=57,763)$ for our main study, results showed significantly lower odds of PTB for midwives' versus GPs' patients [4], suggesting a lack of power to detect differences in effect for PTB within this smaller sample.

Substance using women in the care of midwives versus OBs had 31\% significantly lower odds of PTB compared to non-substance users (aOR 0.24, 95\% CI: 0.11-0.54 versus aOR $0.55,95 \% \mathrm{CI}: 0.45-0.68$ ) (Table 3). Tests showed evidence of effect modification $(p=0.05)$.

For GP versus OB patients, substance use also significantly modified the relationship between antenatal model of care and PTB. GP versus OB patients had 28\% significantly lower odds of PTB compared to non-substance users $(\mathrm{aOR} 0.41,95 \% \mathrm{CI}: 0.28-0.61$ versus aOR $0.69,95 \% \mathrm{CI}: 0.61-0.79)$. GP versus $\mathrm{OB}$ care was 
Table 3 Frequencies and adjusted odds ratios for preterm birth by antenatal model of care and psychosocial risk characteristics, British Columbia, April 1, 2008-December 31, 2012

\begin{tabular}{|c|c|c|c|c|c|c|}
\hline \multicolumn{7}{|c|}{ Preterm birth by model of care } \\
\hline & MW $n=3394$ & GP $n=25,753$ & $\mathrm{OB} n=4746$ & MW vs. GP & MW vs. OB & GP vs. OB \\
\hline & $n(\%)$ & $n(\%)$ & $n(\%)$ & $\mathrm{aOR}(95 \% \mathrm{Cl})$ & $\mathrm{aOR}(95 \% \mathrm{Cl})$ & $\mathrm{aOR}(95 \% \mathrm{Cl})$ \\
\hline \multicolumn{7}{|l|}{ Mental illness ${ }^{a}$} \\
\hline Yes & $56 / 1011(5.54)$ & $344 / 4917(7.00)$ & $71 / 545(13.03)$ & $0.92(0.68-1.24)$ & $0.49(0.34-0.72)$ & ${ }^{*} 0.54(0.41-0.71)$ \\
\hline No & $98 / 2383(4.11)$ & $1257 / 20,836(6.03)$ & $352 / 4201(8.38)$ & $0.73(0.59-0.91)$ & $0.51(0.41-0.65)$ & $0.70(0.61-0.79)$ \\
\hline \multicolumn{7}{|l|}{ Substance use $\mathrm{e}^{\mathrm{b}}$} \\
\hline Yes & $8 / 152(5.26)$ & $215 / 2276(9.45)$ & $39 / 182(21.43)$ & $0.59(0.29-1.21)$ & $* 0.24(0.11-0.54)$ & ${ }^{*} 0.41(0.28-0.61)$ \\
\hline No & $146 / 3242(4.50)$ & $1386 / 23,477(5.90)$ & $384 / 4564(8.41)$ & $0.80(0.67-0.96)$ & $0.55(0.45-0.68)$ & $0.69(0.61-0.79)$ \\
\hline \multicolumn{7}{|l|}{ Teen mother } \\
\hline Yes (14-19yrs.) & 9/117 (7.69) & $187 / 2584(7.24)$ & 21/206 (10.19) & $1.09(0.55-2.19)$ & $0.74(0.33-1.66)$ & $0.67(0.42-1.09)$ \\
\hline No (25-29yrs.) & 46/1161 (3.96) & 475/7705 (6.16) & 100/1371 (7.29) & $0.69(0.50-0.94)$ & $0.56(0.39-0.80)$ & $0.81(0.65-1.02)$ \\
\hline \multicolumn{7}{|l|}{ Social assistance ${ }^{c}$} \\
\hline Yes & $17 / 239(7.11)$ & $299 / 3617(8.27)$ & $61 / 534(11.42)$ & $0.89(0.53-1.50)$ & $0.63(0.36-1.12)$ & $0.71(0.53-0.96)$ \\
\hline No & 137/3155 (4.34) & 1302/22,136 (5.88) & $362 / 4212(8.59)$ & $0.78(0.64-0.94)$ & $0.51(0.42-0.63)$ & $0.66(0.58-0.75)$ \\
\hline \multicolumn{7}{|l|}{ Neigh. SEPd } \\
\hline Low/Medium & $135 / 2944(4.59)$ & $1429 / 22,915(6.24)$ & $397 / 4369(9.09)$ & $0.80(0.66-0.96)$ & $0.52(0.42-0.64)$ & $0.65(0.58-0.74)$ \\
\hline High & $19 / 450(4.22)$ & $172 / 2838(6.06)$ & 26/377 (6.90) & $0.72(0.44-1.17)$ & $0.63(0.34-1.17)$ & $0.88(0.57-1.36)$ \\
\hline \multicolumn{7}{|c|}{ Mental illness, substance use } \\
\hline Both & 5 or less $/ 95^{e}$ & $95 / 927(10.25)$ & 18/76 (23.68) & $0.43(0.15-1.19)$ & ${ }^{*} 0.18(0.06-0.55)$ & ${ }^{*} 0.41(0.23-0.74)$ \\
\hline Neither & $94 / 2325(4.04)$ & $1134 / 19,438(5.83)$ & $331 / 4090(8.09)$ & $0.73(0.59-0.91)$ & $0.52(0.41-0.66)$ & $0.71(0.62-0.81)$ \\
\hline \multicolumn{7}{|c|}{ Mental illness, social assistance } \\
\hline Both & $7 / 128(5.47)$ & 117/1195 (9.79) & 27/161 (16.77) & $0.58(0.26-1.32)$ & $0.35(0.14-0.86)$ & $0.60(0.38-0.96)$ \\
\hline Neither & $88 / 2272(3.87)$ & 1075/18,414 (5.84) & $318 / 3828(8.31)$ & $0.70(0.56-0.88)$ & $0.48(0.37-0.61)$ & $0.68(0.59-0.79)$ \\
\hline \multicolumn{7}{|c|}{ Substance use, social assistance } \\
\hline Both & 5 or less $/ 45^{e}$ & $94 / 832(11.30)$ & 22/93 (23.66) & $0.78(0.27-2.27)$ & $0.34(0.11-1.10)$ & $0.44(0.26-0.76)$ \\
\hline Neither & $133 / 3047(4.36)$ & $1178 / 20,643(5.71)$ & $345 / 4118(8.38)$ & $0.78(0.65-0.95)$ & $0.53(0.43-0.65)$ & $0.67(0.59-0.77)$ \\
\hline
\end{tabular}

Abbreviations: $M W$ midwife, GP general practitioner, $O B$ obstetrician, $O R$ odds ratio, $C l$ confidence interval

${ }^{a}$ Mental illness included any of the following diagnoses prior to, or during the current pregnancy: anxiety disorder, depression, postpartum depression, bipolar disorder, other/unknown (including schizophrenic, mood, and psychotic disorders). Aside from mild anxiety or depression, a physician would diagnose mental illness

${ }^{\mathrm{b}}$ Substance use included any indication in the medical record of heroin/opioids, cocaine, methadone, solvents, marijuana or other/unknown drugs used by the mother at any time during pregnancy, as well as prescription or other drug use identified as a risk by the provider

'Social assistance recipients received public financial assistance during the year of delivery due to low income

${ }^{\mathrm{d}}$ Neighbourhood low/medium socioeconomic position included women residing in the four lowest income quintiles, depending on residential postal code at delivery

'e Percentage suppressed due to small cell size

*Outcomes had statistically significant tests of interaction $(p<0.10)$ comparing difference of effect across strata (yes vs. no)

Models adjusted for all variables listed except stratifying variables: maternal age, medical risk, obstetric risk, pre-pregnancy BMI, infant sex, receipt of social assistance, smoking status, substance use, mental illness/disorder, neighbourhood SEP, Local Health Area socioeconomic rank, and northern residence Odds ratios based on 2178 PTB births and 33,893 total births with no missing information for this analysis

associated with $16 \%$ significantly lower odds of PTB among women with mental illness compared to women without mental illness (aOR 0.54, 95\% CI: 0.41-0.71 versus aOR 0.70, 95\% CI: 0.61-0.79), indicating effect modification.

Midwifery versus $\mathrm{OB}$ patients with both mental illness and substance use had 34\% significantly lower odds of PTB (aOR 0.18, 95\% CI: 0.06-0.55) compared to those with neither risk factor (aOR 0.52, 95\% CI: 0.41-0.66). Comparing GP versus OB patients, odds of PTB were
$30 \%$ significantly lower for those with mental illness and substance use compared to those without these risks (aOR 0.41, 95\% CI: $0.23-0.74$ versus aOR 0.71, 95\% CI: $0.62-0.81)$. None of the psychosocial risk characteristics examined modified the relationship between midwifery versus GP care and PTB (Table 3).

\section{Post-hoc power estimates}

Despite having all population-level data available for eligible midwifery clients in $\mathrm{BC}$ during the study period, 
the sample sizes used to compare modifying factors were small, particularly for the midwifery cohort, potentially impacting the power to detect differences between groups for some outcomes. Power estimations for this study were not conducted prior to the analyses as there have been no published estimates of adverse outcomes among women of low SEP receiving midwifery care in Canada. Post-hoc power estimates indicate low power $(<80 \%$, alpha set at 0.10$)$ for a number of comparisons (Table 4). However, the SGA and PTB analyses were adequately powered to detect differences between midwifery and obstetrician care, modified by mental illness or substance use. Likewise, there was adequate power to assess the association between midwifery versus GP care and SGA, modified by mental illness.

\section{Discussion}

In this study, including only women of low socioeconomic position, the odds of SGA and PTB were lower among those receiving antenatal care from midwives versus $\mathrm{OBs}$ and the association between midwifery care and PTB was stronger among women using substances compared to non-users. Midwifery versus $\mathrm{OB}$ patients with both mental illness and substance use also had lower odds of PTB than those with neither mental illness nor substance use. Comparing antenatal GP versus $\mathrm{OB}$ care, there were lower odds of PTB among GP patients with mental illness, substance use, and combined mental illness and substance use compared to women without these vulnerabilities. The reduction in odds of PTB within subgroups of substance users and substance users with mental health conditions was greatest for midwifery versus $\mathrm{OB}$ patients, followed by GP versus $\mathrm{OB}$ patients. Exposure to the psychosocial risk characteristics examined did not modify the associations between midwifery care and SGA or PTB, compared to GP care.

Previous midwifery/physician studies conducted in high resource countries examining antenatal model of care and poor birth outcomes for women of low SEP have examined only the modifying effect of age [3]. A randomized controlled trial from the USA examined the relationship between low birth weight (LBW) for low SEP women receiving enhanced antenatal care from nurse-midwives versus standard care from $\mathrm{OB}$ residents, stratified by age $(<16,17-19,>20$ years) [21]. Similar to our SGA findings, their results did not indicate a difference in effect in LBW for nurse-midwifery versus physician care by teen maternal age. Likewise, a retrospective cohort study which investigated LBW and very LBW among American Medicaid recipients receiving antenatal nurse-midwifery versus physician care found no difference in relative risk for teen mothers compared to women under 40 [22].

This study was limited to the analysis of variables available in the administrative database. We did not have access to information on mother's race/ethnicity (including Status Indian), education, and measures of maternal health knowledge and attitudes/values. These factors have shown to be important characteristics associated with antenatal model of care and independent risk factors for adverse birth outcomes. However, it should be noted that our sample was restricted to prenatal women with low income (thereby controlling for effects due to $\mathrm{SEP}$ ), and we were able to assess and/or control for

Table 4 Post-hoc power estimates for moderation analyses investigating antenatal models of care by small-for-gestational-age birth and pre-term birth (alpha set at 0.10)

\begin{tabular}{|c|c|c|c|}
\hline Moderating Factor & MW vs. GP (\%) & MW vs. OB (\%) & GP vs. OB (\%) \\
\hline \multicolumn{4}{|l|}{ Small-for-Gestational-Age Birth } \\
\hline Mental illness & 93.96 & 94.63 & 28.17 \\
\hline Substance use & 72.05 & 83.46 & 29.69 \\
\hline Teen mother & 6.43 & 19.80 & 47.04 \\
\hline Mental illness, substance use ${ }^{a}$ & - & - & 18.25 \\
\hline \multicolumn{4}{|l|}{ Preterm Birth } \\
\hline Mental illness & 48.79 & 99.97 & 99.95 \\
\hline Substance use & 47.40 & 99.37 & 99.96 \\
\hline Teen mother & 6.96 & 12.70 & 40.57 \\
\hline Social assistance & 12.50 & 52.39 & 75.36 \\
\hline Neigh. SEP & 96.77 & 100 & 100 \\
\hline Mental illness, substance use ${ }^{a}$ & - & - & 95.83 \\
\hline Mental illness, social assistance & 41.30 & 87.37 & 82.03 \\
\hline Substance use, social assistance ${ }^{a}$ & - & - & 94.62 \\
\hline
\end{tabular}

Abbreviations: $M W$ midwife, GP general practitioner, $O B$ obstetrician, Neigh neighbourhood ${ }^{\text {a }}$ Percentage suppressed due to low frequency of cases 
other factors including smoking and alcohol use during pregnancy, and pre-pregnancy BMI. Our findings were unlikely to be biased by our inability to assess outcomes among women who were Status Indian as they represented approximately $2 \%$ of the female population in $\mathrm{BC}$ during the study period [23].

Despite the WHO endorsing midwifery care as a possible means of reducing the risk of PTB, they acknowledge that the mechanisms linking midwifery care to improved outcomes are currently "unclear" ([2], p90). Likewise, for women of low SEP the pathways between antenatal midwifery care and diminished odds of SGA and PTB are undetermined. However, factors that may positively mitigate the association include improved selfcare (e.g. nutritional habits), increased prenatal care utilization and follow-through on clinical advice, and greater self-efficacy-due to the additional time given at the care encounter, emotional support, and depth of relationship the midwifery model affords. Women of low SEP using substances, or having mental illness and substance use, may struggle with low self-esteem and feelings of worthlessness reflecting their stigmatized social position $[24,25]$. This could affect birth outcomes by impeding motivation for self-care and impacting nutritional habits, prenatal care utilization, and follow-through on clinical advice $[26,27]$. The explicit emphasis on dignity and empowerment within the midwifery model [28] may help to dispel unhealthy self-concepts and expectations of clinician judgement, frequently reported by individuals of low SEP [29], thus lowering the prevalence of higher risk behaviour (e.g. self-medicating). The midwifery model encourages women to maintain "agency" described as the autonomy and empowerment that exist when women retain control as the primary-decision makers in their own care ([30], p26). Promoting informed choice, midwives work "in partnership with women to strengthen women's own capabilities to care for themselves and their families" ([31], p3). By facilitating ownership over pregnancy, health, and lifestyle choices, the midwifery model may foster self-efficacy-the belief that one has the ability to effect personal change or reach goals $[32,33]-$ a pre-cursor to cessation or reduction of high risk behaviour (e.g. substance use).

Interestingly, there was higher prevalence of mental health conditions recorded in the maternity record among midwives' clients (29.79\%) compared to physicians' patients (GP 19.09\%; OB 11.46\%). This may be because midwifery clients were more willing to disclose mental health concerns due to greater clinician-client trust cultivated through continuity of care and longer appointments. Or, midwives may have practiced more holistic care (e.g. inquiring about emotional well-being throughout pregnancy) and have had greater opportunity for clinical observation. If mental health diagnosis was greater among midwifery clients due to the antenatal care model, then midwifery care may have been more conducive to the prevention of infant morbidity through mental health treatment, explaining in part the lower odds of PTB for midwifery versus obstetrician patients with psychosocial risk.

\section{Conclusion}

Among low-income prenatal women, those experiencing psychosocial risk including substance use, or mental illness and substance use, have lower rates of PTB if receiving midwifery versus $\mathrm{OB}$ care. A strength of this study is that it explicitly highlights differences in birth outcomes among women with low SEP and other psychosocial risk attributes, by model of care. However, more research is needed to determine how differing antenatal models of care impact women's and newborn's health. Future studies should examine aspects of antenatal maternity models that diverge (e.g. length of appointments, emotional support, mental health screening, behavioural/nutritional counselling, self-care and empowerment education) and how these factors contribute to variation in outcomes. In addition, further insight may be gleaned from a comparison of characteristics of midwifery and GP models of care that coincide but differ from $\mathrm{OB}$ care as results indicated similarity in outcomes among midwives and GPs in contrast to OBs. Our study should be replicated with larger midwifery samples to further explore modification of effects within strata of psychosocial risk. This could further provide guidance on strategies for enhancing and tailoring prenatal care according to women's specific needs.

\section{Additional file}

Additional file 1: Sensitivity Analyses. This file (appendix_a_sensitivity analyses.pdf) contains results of two sensitivity analyses, displayed in Table 1: Adjusted odds ratios and $95 \%$ Cls without and with control for antepartum morbidity and Table 2: Adjusted odds ratios and $95 \% \mathrm{Cls}$ for full study population and for study population excluding pregnancies in which mothers had medical risk or prior obstetric risk. (DOCX 39 kb)

\section{Abbreviations \\ aOR: Adjusted odds ratio; BC Stats: British Columbia's Division of Statistics; BC: British Columbia; BMl: Body mass index; GEE: Generalized estimating eq.; GP: General practitioner; HIV: Human immunodeficiency virus; ICD-10- CA: International Statistical Classification of Diseases, Tenth Revision, Canada; LBW: Low birth weight; LHA: Local Health Area; MSP: Medical Services Plan; MW: Midwife/midwifery; OB: Obstetrician; PDR: Perinatal Data Registry; PTB: Preterm birth; SEP: Socioeconomic position; SGA: Small-for-gestational- age birth; USA: United States of America; WHO: World Health Organization}

\section{Acknowledgements}

We thank Celeste Bickford for assistance in linking the Research Extracts and data preparation.

Disclaimers

All inferences, opinions, and conclusions drawn in this study are those of the authors, and do not reflect the opinions or policies of the Data Stewards. 


\section{Authors' contributions}

DNM designed the study, conducted the statistical analyses, interpreted the results, drafted the initial manuscript, and revised subsequent drafts. NM and PAJ designed the study, reviewed the statistical analyses, interpreted the results, and reviewed and revised the manuscript.

\section{Funding}

DNM was supported by an Arthur Smyth Scholarship from the College of Medicine and a PhD Scholarship from the Department of Community Health and Epidemiology, University of Saskatchewan. PAJ is supported by a Senior Scholar salary award from the BC Children's Hospital Research Institute. Funding sources had no involvement in the study; the authors are independent of all funders.

\section{Availability of data and materials}

No additional data available due to confidentiality restrictions.

\section{Ethics approval and consent to participate}

Ethical approval for this study was granted by the University of Saskatchewan, Biomedical Research Ethics Board (registration numbers 00001471 and 00008358) and the University of British Columbia, Children's and Women's Health Center of BC Research Ethics Board (registration number H14-01629). This study relied on administratively collected data that was anonymized prior to release to the researchers. Participant consent was not required.

\section{Consent for publication}

Not applicable.

\section{Competing interests}

From May 2018 to January 2019 DNM providing consulting services to the Midwives Association of British Columbia. No other authors have competing interests to declare.

\section{Author details}

${ }^{1}$ Department of Community Health and Epidemiology, University of Saskatchewan, Box 7 Health Science Building 107 Wiggins Road, Saskatoon SK S7N 5E5, Canada. ${ }^{2}$ School of Population and Public Health, University of British Columbia, 2206 East Mall, Vancouver BC V6T 1Z3, Canada.

Received: 19 March 2019 Accepted: 25 July 2019

Published online: 06 August 2019

\section{References}

1. Sandall J, Soltani H, Gates S, Shennan A, Devane D. Midwifery-led continuity models versus other models of care for childbearing women. Cochrane Database Syst Rev. 2016;(4):CD004667. https://doi.org/10.1002/14651858. CD004667.pub5.

2. World Health Organization. WHO recommendations on antenatal care for a positive pregnancy experience. Geneva: WHO; 2016 [cited 2019 Jan 18]. Available from: http://apps.who.int/iris/bitstream/handle/10665/250796/ 9789241549912-eng.pdf?sequence $=1$.

3. McRae DN, Muhajarine N, Stoll K, et al. Is model of care associated with infant birth outcomes among vulnerable women? A scoping review of midwifery-led versus physician-led care. Soc Sci Med-Pop Health. 2016;2: 182-93. https://doi.org/10.1016/j.ssmph.2016.01.007.

4. McRae DN, Janssen PA, Vedam S, et al. Reduced prevalence of small-forgestational-age and preterm birth for women of low socioeconomic position: a population-based cohort study comparing antenatal midwifery and physician models of care. BMJ Open. 2018;8(10). https://doi.org/1 0.1136/bmjopen-2018-022220.

5. College of Midwives of British Columbia. Indications for Discussion, Consultation and Transfer of Care. CMBC; 2018 [cited 2017 May 2]. Available from: https://www.cmbc.bc.ca/wp-content/uploads/2018/05/Indications-forDiscussion-Consultation-and-Transfer-of-Care.pdf.

6. College of Midwives of British Columbia (CMBC). Midwifery Model of Practice: CMBC; 2013 [cited 2017 April 22]. Available from: http://www.cmbc. bc.ca/pdf.shtml? Registrants-Handbook-11-05-midwifery-model-of-practice.

7. Vedam S, Stoll K, Martin K, et al. The Mother's autonomy in decision making (MADM) scale: patient-led development and psychometric testing of a new instrument to evaluate experience of maternity care. PLoS One. 2017;12(2). https://doi.org/10.1371/journal.pone.0171804.
8. Perinatal Services BC [creator] (2016): British Columbia perinatal data registry. Population data BC [publisher]. Data extract. PSBC (2014) [cited April 6]. Available from: http://www.perinatalservicesbc.ca/healthprofessionals/data-surveillance/perinatal-data-registry.

9. Perinatal Services BC (PSBC). BC Perinatal Data Registry (PDR) Data Access Requests (DAR) for Research Purposes (non-Popdata Projects). Frequently Asked Questions V.1.5. Vancouver, BC: PSBC; 2017 [cited 2019 July 11]. Available from: http://www.perinatalservicesbc.ca/Documents/DataSurveillance/PDR/DataRequests/DAR_FAQ.pdf.

10. Frosst G, Hutcheon J, Joseph KS, Kinniburgh B, Johnson C, Lee L. Validating the British Columbia perinatal data registry: a chart re-abstraction study. BMC Pregnancy Childbirth. 2015;15:123. https://doi.org/10.1186/s12884-015-0563-7.

11. British Columbia Ministry of Health [creator]. Medical Services Plan (MSP) Payment Information File. V2. Data Extract. MOH (2014) Population Data BC [publisher]; 2016 [cited 2017 April 6]. Available from: http://www.popdata. bc.ca/data

12. British Columbia Ministry of Health [creator]. Consolidation file (MSP Registration \& Premium Billing). Population data BC [publisher]. Data extract. MOH (2014); 2015. [cited 2017 April 6]. Available from: http://www.popdata. bc.ca/data.

13. B.C. Stats. Socio-economic indices: overall regional socio-economic index: Government of B.C.; 2013 [cited 2014 Nov 4]. Available from: http://www. bcstats.gov.bc.ca/StatisticsBySubject/SocialStatistics/

SocioEconomicProfilesindices/SocioEconomicindices/LHAReports.aspx.

14. B.C. Stats. Socio-economic indices: LHA indices reports. Human economic hardship: income inequality measure: Government of B.C.; 2013 [cited 2014 Nov 4]. Available from: http://www.bcstats.gov.bc.ca/StatisticsBySubject/ SocialStatistics/SocioEconomicProfilesIndices/SocioEconomiclndices/ LHAReports.aspx.

15. Government of Canada. What is Indian status? Ottawa: Government of Canada; 2018 [cited 2019 June 30]. Available from: https://www.aadncaandc.gc.ca/eng/1100100032463/1100100032464.

16. Kierans WJ, Kendall P, Foster LT, Liston RM, Tuk T. New birth weight and gestational age charts for the British Columbia population. BCMJ. 2006;48(1): $28-32$.

17. SAS Institute Inc. SAS/STAT ${ }^{\circledR} 14.3$ User's Guide: The GEE procedure. Cary, NC: SAS Institute Inc:; 2017 [cited 2016 Dec 1]. Available from: http://support.sas. com/documentation/onlinedoc/stat/143/gee.pdf.

18. Greenland S. Interactions in epidemiology: relevance, identification, and estimation. Epidemiol. 2009;20(1):14-7. https://doi.org/10.1097/EDE.0b013 e318193e7b5.

19. Bloom HS, Michalopoulos C. When is the story in the subgroups? Prev Sci. 2013;14:179-88. https://doi.org/10.1007/s11121-010-0198-X.

20. SAS Institute Inc. SAS Enterprise 7.1 Cary: SAS Institute Inc.

21. McLaughlin FJ, Altemeier WA, Christensen MJ, Sherrod KB, Dietrich MS, Stern DT. Randomized trial of comprehensive prenatal care for low-income women: effect on infant birth weight. Pediatrics. 1992;89(1):128-32.

22. Visintainer $P$, Uman J, Horgan D, Ibald A, Verma U, Tejani N. Reduced risk of low weight births among indigent women receiving care from nursemidwives. J Epidemiol Community Health. 2000;54(3):233-8. https://doi. org/10.1136/jech.54.3.233.

23. Statistics Canada. Aboriginal peoples: fact sheet for British Columbia. Ottawa: Stats Can; 2016 [cited 2018 Jan 25]. Available from: http://www. statcan.gc.ca/pub/89-656-x/89-656-x2016011-eng.htm.

24. Livingston JD, Boyd JE. Correlates and consequences of internalized stigma for people living with mental illness: a systematic review and meta-analysis. Soc Sci Med. 2010;71(12):2150-61. https://doi.org/10.1016/j.socscimed.2010.09.030.

25. Livingston JD, Milne T, Fang ML, Amari E. The effectiveness of interventions for reducing stigma related to substance use disorders: a systematic review. Addiction. 2012;107(1):39-50. https://doi.org/10.1111/ j.1360-0443.2011.03601.x.

26. Grote NK, Bridge JA, Gavin AR, Melville JL, lyengar S, Katon WJ. A metaanalysis of depression during pregnancy and the risk of preterm birth, low birth weight, and intrauterine growth restriction. Arch Gen Psychiatry. 2010; 67(10):1012-24. https://doi.org/10.1001/archgenpsychiatry.2010.111.

27. Rubio DM, Kraemer KL, Farrell MH, Day NL. Factors associated with alcohol use, depression, and their co-occurrence during pregnancy. Alcohol Clin Exp Res. 2008;32(9):1543-51. https://doi.org/10.1111/j.1530-0277.2008.00705.x.

28. College of Midwives of British Columbia (CMBC). Philosophy of Care. CMBC; 2019 [cited 2019 July 31]. Available from: https://www.bcmidwives.com/ philosophy_care.html. 
29. Arpey NC, Gaglioti AH, Rosenbaum ME. How socioeconomic status affects patient perceptions of health care: a qualitative study. J Prim Care Community Health. 2017:8(3):169-75. https://doi.org/10.1177/215 0131917697439.

30. Devane D, Brennan M, Begley C, et al. Socioeconomic value of the midwife: a systematic review, meta-analysis, meta-synthesis and economic analysis of midwife-led models of care. London, U.K.: Royal College of Midwives; 2010 [cited 2017 March 16]. Available from: https://pdfs.semanticscholar.org/2 eb1/de2ba0a8fe1db27506c1b515578f75e44fb2.pdf.

31. Renfrew MJ, Homer C, Downe S, et al. Midwifery. An executive summary for the Lancet's series. Lancet. 2015;384:9948.

32. Van Herk KA, Smith D, Andrew C. Identity matters: aboriginal mothers' experiences of accessing health care. Contemp Nurse. 2010;37(1):57-68. https://doi.org/10.5172/conu.2011.37.1.057.

33. Smith DA, Edwards NC, Martens PJ. Varcoe C. 'making a difference': a new care paradigm for pregnant and parenting aboriginal people. Can J Public Health. 2007;98(4):321-5. https://doi.org/10.17269/cjph.98.881.

34. Health Canada. Prenatal nutrition guidelines for health professionals: gestational weight gain. Ottawa: Health Canada; 2014 [cited 2017 Nov 9]. Available from: http://www.hc-sc.gc.ca/fn-an/nutrition/prenatal/ewba-mbsaeng.php\#t2.

\section{Publisher's Note}

Springer Nature remains neutral with regard to jurisdictional claims in published maps and institutional affiliations.

Ready to submit your research? Choose BMC and benefit from:

- fast, convenient online submission

- thorough peer review by experienced researchers in your field

- rapid publication on acceptance

- support for research data, including large and complex data types

- gold Open Access which fosters wider collaboration and increased citations

- maximum visibility for your research: over $100 \mathrm{M}$ website views per year

At BMC, research is always in progress.

Learn more biomedcentral.com/submissions 